\title{
Proyecto para la mejora de la competitividad comercial del hotel allegro palma real
}

Project to improve the commercial competitiveness of the Allegro Palma Real hotel

Bisleivys Jiménez Valero. ${ }^{1}$, Elianay Piñeyro Alemán. ${ }^{2}$, Roxana B. Hernández Lorenzo. ${ }^{3}$, \& María Laura Pérez Socorro. ${ }^{4}$

\begin{abstract}
.
DOI: https://doi.org/10.33262/visionariodigital.v4i4.1438

The investigation is carried out in the hotel Allegro Real Palm, it has as general objective: to elaborate a project of improvement of the commercial competitiveness of this installation. Theoretical and empiric methods were used among those that stand out: the Historical one - logical and analytic - synthetic, the revision of documental sources (theoretical Level) and the field work, direct observation, storm of ideas and the interview (empiric Level). The application of the Integrated Management of Projects to the solution of commercialization's problems, through viable tools, inside those that stand out: analysis of the situation, of the problems, of the objectives and prefactibilidad study, allows as a result to elaborate a plan of improvements for the competitive situation of the hotel.
\end{abstract}

Keywords: Commercial competitiveness, Integrated Management of Projects, investigation project, hotel, commercial improvements.

\section{Resumen.}

La investigación se realiza en el hotel Allegro Palma Real, tiene como objetivo general: elaborar un proyecto de mejora de la competitividad comercial de dicha instalación. Se emplearon métodos teóricos y empíricos entre los que se destacan: el histórico- lógico y analítico- sintético, la revisión de fuentes documentales (nivel teórico) y el trabajo de campo, observación directa, tormenta de ideas y la entrevista semiestructurada (nivel empírico). La aplicación de la Dirección Integrada de Proyectos a la solución de problemas de comercialización, a través de viables herramientas, dentro de las que se destacan: análisis de la situación, de los problemas, de los objetivos y estudio de

\footnotetext{
${ }^{1}$ Universidad de Matanzas, Matanzas, Cuba, bisleivys.jimenez@umcc.cu.

${ }^{2}$ Universidad de Matanzas, Matanzas, Cuba, ely.ely@nauta.cu.

${ }^{3}$ Universidad de Matanzas, Matanzas, Cuba, bisleivys.jimenez@umcc.cu.

${ }^{4}$ Universidad de Matanzas, Matanzas, Cuba, bisleivys.jimenez@umcc.cu.
} 
prefactibilidad, permite como resultado elaborar un plan de mejoras para la situación competitiva del hotel.

Palabras claves: Competitividad comercial, Dirección Integrada de Proyectos, proyecto de investigación, hotel, mejora comercial.

\section{Introducción.}

El turismo constituye un sector clave para el progreso socioeconómico, a través de la creación de puestos de trabajo y de empresas, la generación de ingresos de exportación y la ejecución de infraestructuras. En la última década esta actividad ha experimentado una continua expansión y diversificación, convirtiéndose en uno de los sectores económicos de mayor envergadura y crecimiento del mundo (Rodríguez, 2015).

El sector turístico es cada vez más exigente, por lo que requiere de prácticas de dirección y gestión más integrales para su funcionamiento óptimo. En este sentido la dirección integrada de proyecto constituye una alternativa factible a la solución de problemas de diversa índole en la industria sin humo, donde cobran relevante interés las viables estrategias de comercialización, como elemento clave para el éxito empresarial (Hernández, 2016).

Varadero, ubicado en la provincia de Matanzas, se destaca como uno de los principales destinos turísticos de Cuba. Dentro de sus ofertas se encuentra el hotel Allegro Palma Real, perteneciente al grupo hotelero Gran Caribe, opera en contrato de administración con la cadena Barceló. Se sitúa en la zona conocida como el centro de la recreación del destino (calle 62 e/1ra y 2 da avenida) y a 25 minutos de distancia del aeropuerto internacional de Varadero Juan Gualberto Gómez.

Según informe del departamento comercial del hotel Allegro Palma Real (2018) se ha hecho notable la presencia de comentarios desfavorables en las redes sociales, así como la determinación mediante las encuestas de fronteras (aplicadas a partir del año 2015) de una marcada insatisfacción, relacionada con los servicios y procesos de la entidad, lo cual influye en costos de no calidad y compensaciones a clientes. Dicha situación perjudica el estado comercial del hotel y su posicionamiento en el mercado.

Existen algunas investigaciones antecedentes que abordan la Dirección Integrada y Evaluación de proyectos y la competitividad comercial, indistintamente, díganse en tal 
sentido: Pérez (2008); Méndez (2015); Guerrero (2013); Hernández (2016); Castillo (2017); Serrano (2018) sin embargo son escasos los estudios precedentes que vinculen los proyectos a la competitividad comercial.

A partir de la problemática planteada se define el siguiente problema científico:

¿Cómo contribuir a la mejora de la competitividad comercial en el Hotel Allegro Palma Real?

En correspondencia con el problema definido, se traza como Objetivo General: Elaborar un proyecto que contribuya a la elevación de la competitividad comercial en el Hotel Allegro Palma Real.

Del objetivo general se desprenden como objetivos específicos:

1. Fundamentar los referentes teórico-metodológicos de la investigación relacionados con los Proyectos, la Dirección Integrada de Proyectos, la comercialización y la competitividad comercial hotelera.

2. Elaborar un procedimiento para la mejora de la competitividad comercial en un hotel.

3. Proponer un plan de mejoras para la competitividad comercial de la instalación hotelera objeto de estudio.

Para el desarrollo de la investigación se han aplicado diferentes métodos teóricos y empíricos:

Nivel teórico: Histórico- lógico y analítico- sintético. Revisión de fuentes documentales como revistas, artículos científicos, tesis de diploma y maestría.

Nivel empírico: Trabajo de campo, observación directa, tormenta de ideas para diagnosticar el objeto de estudio y entrevista semiestructurada al director comercial de la instalación.

Se han utilizado herramientas de la Dirección Integrada de Proyectos para la solución de problemas de comercialización, como: análisis de la situación, de los involucrados, de los problemas, de los objetivos, estudio de prefactibilidad, matriz de marco lógico, matriz de funciones/responsabilidades y estructura fraccionada del trabajo. 
ISSN: 2602-8506

Vol. 4, $\mathrm{N}^{\circ} 4$, p. 48-65, octubre-diciembre, 2020

El resultado esperado es la elaboración de un plan de mejoras para la situación competitiva del hotel.

Figura 1: Procedimiento metodológico de la investigación

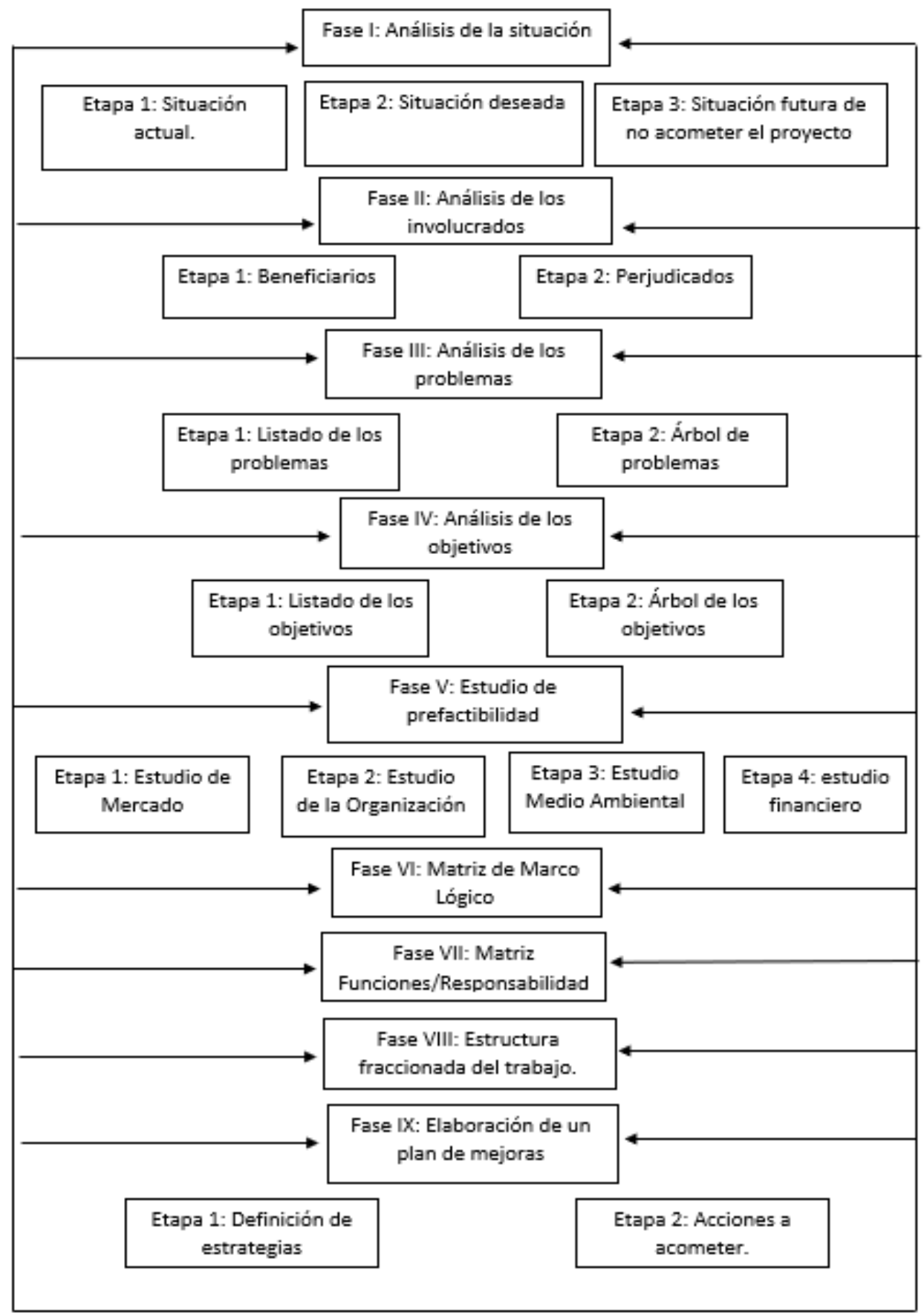

Fuente: Elaboración propia. 


\section{Resultados.}

En el presente capítulo se exponen los resultados obtenidos a través de la aplicación del procedimiento esquematizado anteriormente:

\section{Análisis de la situación:}

- El hotel Allegro Palma Real opera en contrato de administración con la Cadena Barceló, bajo la modalidad de todo incluido y pertenece al grupo hotelero de Gran Caribe.

- Se sitúa en la zona conocida como el centro de la recreación del destino Varadero (calle 62 e/1ra y 2 da avenida).

- Se encuentra a 25 minutos de distancia del Aeropuerto Internacional de Varadero Juan Gualberto Gómez.

- En su infraestructura arquitectónica posee 4 edificios de alojamiento, desde 5 pisos con169 habitaciones cada uno y dos módulos habitacionales de 2 pisos y 64 habitaciones cada uno.

- La instalación cuenta con una casa en la playa de 4 habitaciones para un total de 470 habitaciones.

- Las habitaciones son estándar, todas con baño privado, aire acondicionado, TV vía satélite, teléfono, caja de seguridad y corriente 220v. Poseen un balcón con vista a la piscina, o a la costa sur de la península.

- La instalación tiene 3 piscinas para adultos y 1 para niños, así como un teatro para espectáculos con capacidad para 320 clientes.

- Dentro de las facilidades para el servicio gastronómico existe un restaurante buffet para 330 comensales, 3 restaurantes a la carta, especializados en distintos tipos de cocinas, con una capacidad total de 176 clientes; cinco bares con servicios que abarcan entre 8,12 y 24 horas y un snack bar abierto 24 horas.

- Las encuestas de satisfacción realizadas por el departamento de calidad del hotel a clientes en el año 2018 arrojan en promedio un $20 \%$ de insatisfacción con los servicios y procesos de la entidad, lo que repercute en costos de no calidad y compensaciones a clientes. 
- El $20 \%$ del mobiliario se encuentra deteriorado por el tiempo prolongado de explotación en habitaciones y áreas públicas.

- Inestabilidad de los suministros y abastecimientos durante todo el año fundamentalmente abril - agosto.

- Existen problemas con algunos marcos y puertas de entradas y ventanas interiores de habitaciones que necesitan reparación.

- Equipamiento obsoleto e insuficiente en los sistemas de Bombeo y Cocina.

- Sistema de agua caliente inestable.

- Ausencia prolongada de Ascensores de Clientes.

- Piscinas muy deterioras y con serias afectaciones tecnológicas.

- Alta dependencia de los turoperadores.

- Limitadas estrategias comerciales (las existentes giran fundamentalmente en torno a la consolidación de los mercados tradicionales de la instalación, se otorga poca importancia a la atracción de nuevos mercados potenciales).

\section{Situación deseada.}

Alcanzar un posicionamiento en el Trip advisor inferior al 20 para mejorar la situación competitiva del hotel. Tener clientes más complacidos y fieles a la instalación y disminuir los costos de no calidad por las compensaciones.

Situación futura de no acometer el proyecto

Posicionamiento en el trip advisor superior a 20; aumento de la insatisfacción de los clientes; incremento de los costos de no calidad, lo que agravaría la situación competitiva.

\section{Análisis de involucrados.}

En el proyecto se involucran varias personas y empresas, algunos son beneficiarios (clientes externos, trabajadores de la entidad, MINTUR, Grupo Hotelero Gran Caribe, la cadena Barceló) y otros son perjudicados como la competencia (hoteles del destino Varadero de igual categoría o pertenecientes al mismo grupo hotelero. 


\section{Análisis de problemas.}

- Limitadas estrategias comerciales.

- Índice de satisfacción de los clientes inferior al $80 \%$ y elevados costos de no calidad.

- Mal estado de las instalaciones del hotel.

- Dificultad para atraer nuevos mercados.

- Daño a la imagen del hotel.

Problema focal: Insuficiente competitividad comercial del Hotel Allegro Palma Real, que se evidencia en el desventajoso posicionamiento en el Trip Advisor (se ha situado por encima del lugar 25 con respecto a los hoteles del destino Varadero en el año 2018)

\section{Árbol de problemas:}

Figura 2: Árbol de problemas

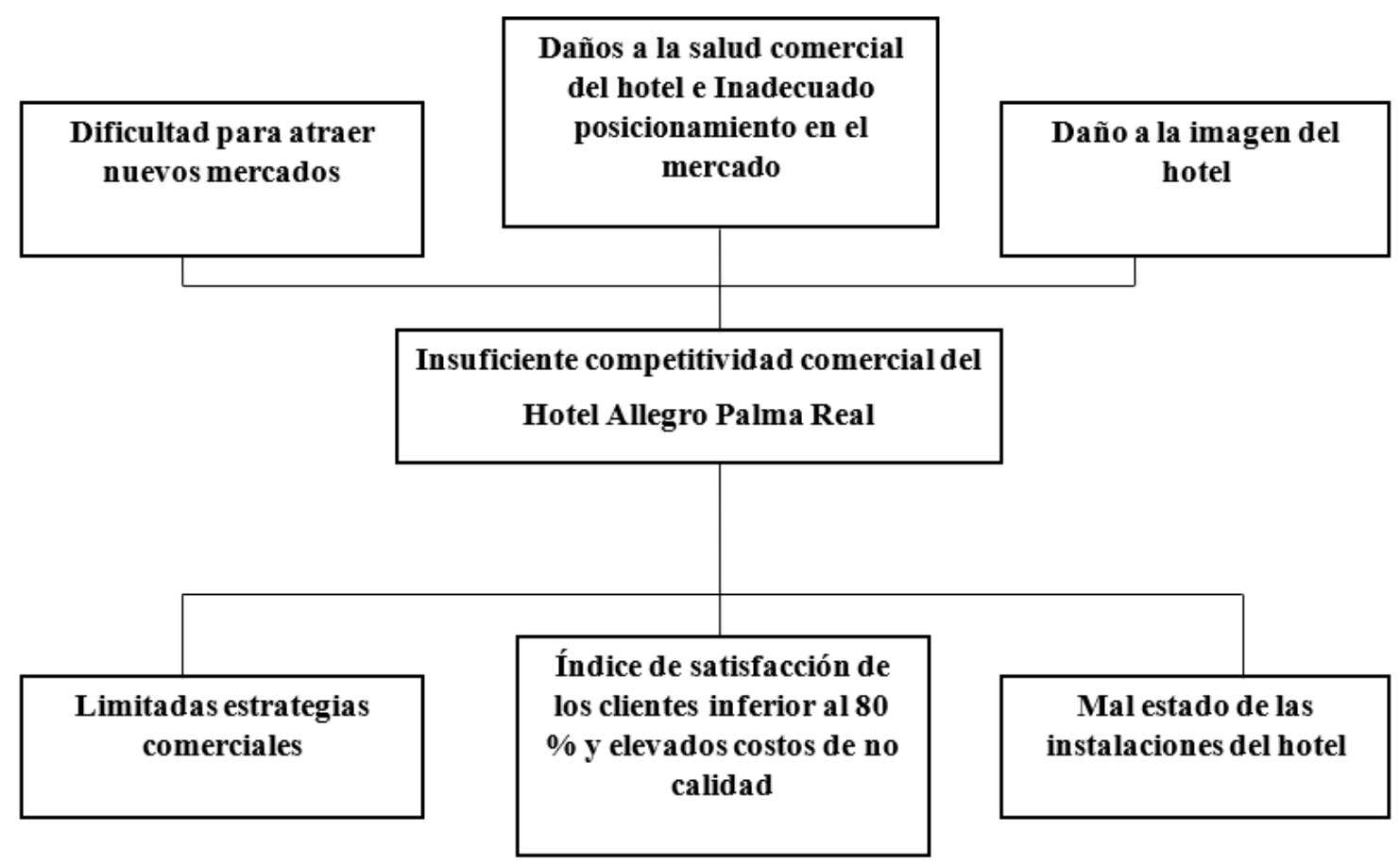

Fuente: Elaboración propia. 


\section{Análisis de objetivos.}

Objetivo focal: Elevar en un 5\% la competitividad comercial del Hotel Allegro Palma Real.

Figura 3: Árbol de objetivos.

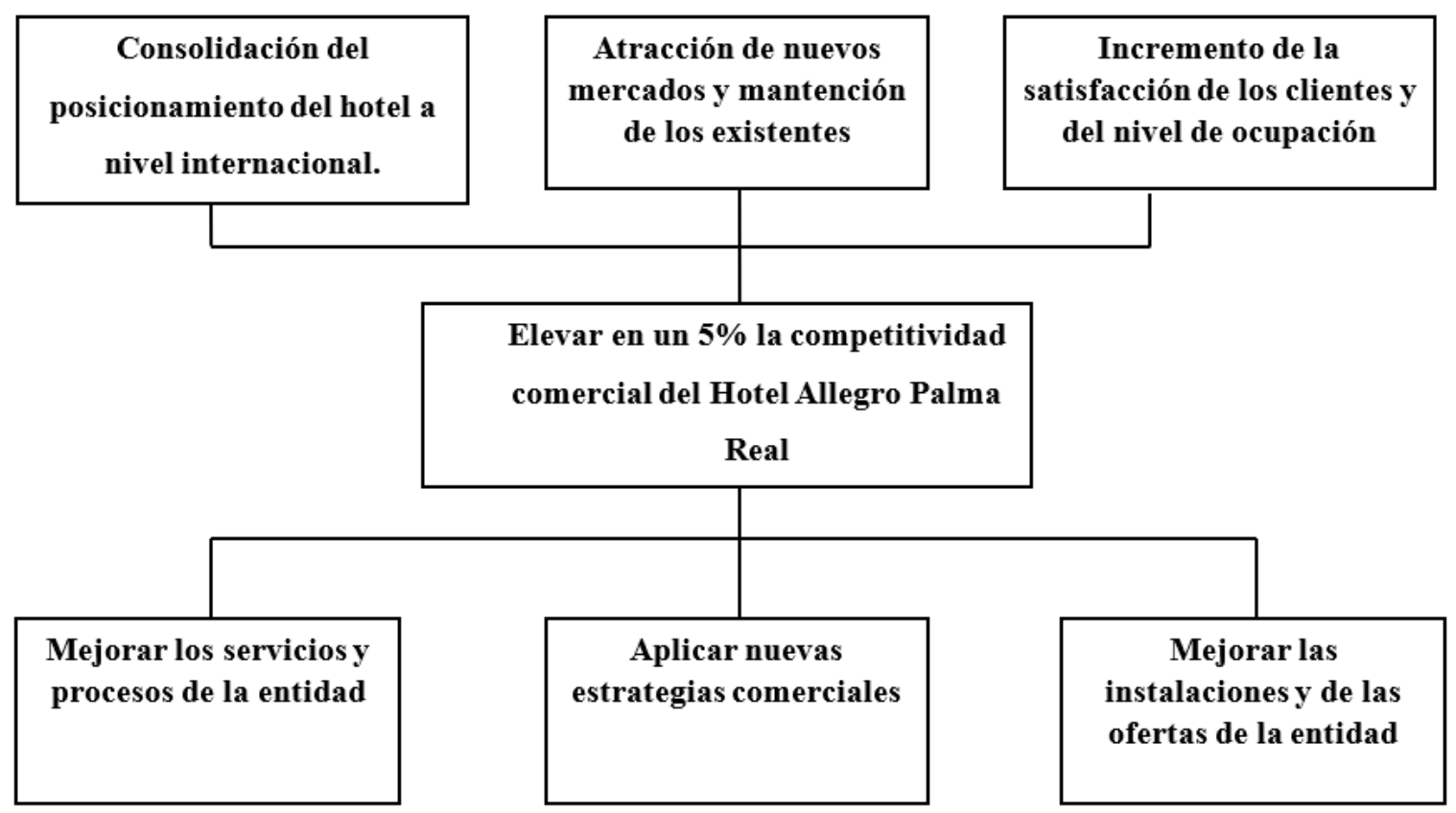

Fuente: Elaboración propia.

\section{Alternativas:}

A. Elevar la competitividad comercial del hotel Allegro Palma Real para mejorar su posicionamiento en el mercado.

B. Insertar nuevas estrategias comerciales para atraer otros mercados y lograr la fidelización de los ya existentes.

C. Lograr mayor satisfacción de los clientes para disminuir costos de no calidad.

\section{Estudios de prefactibilidad.}

\section{De Mercado:}

- Demanda inicial probable de nuevos mercados a través de turoperadores y agentes de viajes de este mercado. 
- Hotel de sol y playa atractivo, con elementos de cubanía (promoción del ritmo salsero).

\section{Riesgos:}

La calidad de la infraestructura y los servicios no esté acorde con los objetivos comerciales y con la imagen a ofrecer a los clientes.

\section{Organizacional:}

- Necesidad de mejorar la planta hotelera.

- Necesidad de redefinir estrategias para las ofertas hacia los diferentes mercados.

\section{Riesgos:}

No disponer de un personal capacitado para dirigir adecuadamente la comercialización de la instalación.

\section{Medio Ambiental:}

- Entorno de altos valores como producto turístico de sol y playa.

- Ofertas que se consideran adecuadas al entorno.

\section{Riesgos:}

- Elevar demasiado los niveles de comercialización que rebase la capacidad de carga del área de playa.

\section{Financiera:}

- Bajos ingresos, por concepto de comercialización.

- Bajo nivel de presupuesto para el mantenimiento de las habitaciones.

\section{Riesgos:}

- No disponer de un marco financiero inicial para la reapertura o lanzamiento hacia los mercados. 


\section{Comportamiento del flujo de cajas.}

\begin{tabular}{|c|c|c|c|}
\hline Años/proyectos & $\mathbf{0}$ & $\mathbf{1}$ & $\mathbf{2}$ \\
\hline A & $\mathbf{- \$ 3 5 0 0}$ & $\mathbf{\$ 3 0 0 0}$ & $\mathbf{\$ 2 5 0 0}$ \\
\hline B & $-\$ 3500$ & $\$ 2000$ & $\$ 1500$ \\
\hline C & $-\$ 3500$ & $\$ 2000$ & $\mathbf{\$ 1 0 0 0}$ \\
\hline
\end{tabular}

\section{Cálculo del VAN}

Fórmula. VAN $=-\operatorname{Inv} \pm \sum$ VF $1 /(1+\mathrm{k}) \mathrm{n} \quad \mathrm{k}=0.10$

\section{Proyecto A}

$\mathrm{VAN}=-\$ 3500+\$ 3000 \times 1 /(1+0.10)+\$ 2500 \times 1 /(1+0.10) 2$

$\mathrm{VAN}=-\$ 3500+\$ 3000 \times 1 /(1.10)+\$ 2500 \times 1 /(1,21)$

VAN $=-\$ 3500+\$ 3000 \times 0.9090+\$ 2500 \times 0,8264$

$\mathrm{VAN}=-\$ 3500+\$ 2727+\$ 2066$

$\mathrm{VAN}=\$ 1293$

Proyecto B

$\mathrm{VAN}=-\$ 3500+\$ 2000 \times 1 /(1+0.10)+\$ 1500 \times 1 /(1+0.10) 2$

$\mathrm{VAN}=-\$ 3500+\$ 2000 \times 1 /(1.10)+\$ 1000 \times 1 /(1,21)$

$\mathrm{VAN}=-\$ 3500+\$ 2000 \times 0.9090+\$ 1000 \times 0.8264$

$\mathrm{VAN}=-\$ 3500+\$ 1818+\$ 826.4$

$\mathrm{VAN}=-\$ 855.6$

Proyecto C

$\mathrm{VAN}=-\$ 3500+\$ 2000 \times 1 /(1+0.10)+\$ 1500 \times 1 /(1+0.10) 2$

VAN $=-\$ 3500+\$ 2000 \times 1 /(1.10)+\$ 1500 \times 1 /(1,21)$

$\mathrm{VAN}=-\$ 3500+\$ 2000 \times 0.9090+\$ 1500 \times 0.8264$ 
$\mathrm{VAN}=-\$ 3500+\$ 1818+\$ 1239.6$

$\mathrm{VAN}=-\$ 44.24$

Es factible económicamente llevar a cabo el proyecto A porque al calcular el VAN se obtiene $\$ 1293$, o sea un resultado mayor que 0 , por lo que presenta el mayor valor presente de los rendimientos futuros, descontados al costo de capital apropiado, menos el costo de la inversión.

Matriz de Riesgo/niveles

\begin{tabular}{|l|c|c|c|c|c|}
\hline & Mercado & Técnica & Financiera & Organizacional & Medio Ambiental \\
\hline Finalidad & & & & \\
\hline Objetivos & Resultados & & & & \\
\hline Insumos & S & & & \\
\hline
\end{tabular}

Manejo de Riesgos

\begin{tabular}{|c|c|c|}
\hline Tipo de Riesgo & Consecuencias & Acciones a Acometer \\
\hline Mercado & $\begin{array}{l}\text { Disminución de la demanda de } \\
\text { turistas a través } \\
\text { turoperadores y agencias } \\
\text { viajes. }\end{array}$ & $\begin{array}{l}\text { Mejorar la calidad de la } \\
\text { infraestructura y los servicios y } \\
\text { desarrollar estrategias comerciales de } \\
\text { acuerdo a los objetivos. }\end{array}$ \\
\hline Organizacional & $\begin{array}{lrr}\text { Disminución } & \text { de } & \text { la } \\
\text { comercialización } & \text { de } & \text { la } \\
\text { instalación } & & \\
\end{array}$ & $\begin{array}{l}\text { Capacitar al personal vinculado a la } \\
\text { actividad comercial }\end{array}$ \\
\hline Financiera & $\begin{array}{l}\text { Dificultad para llevar a cabo } \\
\text { estrategias de comercialización } \\
\text { y promoción. }\end{array}$ & $\begin{array}{l}\text { Incrementar los ingresos a través de } \\
\text { nuevas ofertas. }\end{array}$ \\
\hline Medioambiental & $\begin{array}{l}\text { Impacto en el área de playa del } \\
\text { hotel debido a la sobrecarga. }\end{array}$ & $\begin{array}{l}\text { Planificar adecuadamente la } \\
\text { comercialización de la instalación en } \\
\text { correspondencia con la capacidad de } \\
\text { carga. }\end{array}$ \\
\hline
\end{tabular}

Fuente: Elaboración propia. 


\section{Matriz de Marco Lógico:}

\begin{tabular}{|c|c|c|c|c|}
\hline & Resumen Narrativo & Indicadores & $\begin{array}{c}\text { Medios de } \\
\text { Verificación }\end{array}$ & Condiciones Críticas \\
\hline Finalidad & $\begin{array}{l}\text { Alcanzar un } \\
\text { posicionamiento en el } \\
\text { Trip advisor inferior al } 20 \\
\text { para mejorar la situación } \\
\text { competitiva del hotel. } \\
\text { Tener clientes más } \\
\text { complacidos y fieles a la } \\
\text { instalación y disminuir } \\
\text { los costos de no calidad } \\
\text { por las compensaciones. }\end{array}$ & $\begin{array}{l}\text { Papel de la competencia. } \\
\text { Papel de los } \\
\text { intermediarios. } \\
\text { Evaluación de las } \\
\text { condiciones del hotel. } \\
\text { Evaluación de la demanda } \\
\text { actual y futura. }\end{array}$ & $\begin{array}{l}\text { Balance Económico } \\
\text { Balance Comercial }\end{array}$ & $\begin{array}{l}\text { Aumento del deterioro de } \\
\text { las instalaciones, ofertas y } \\
\text { servicios de la entidad. } \\
\text { Aumento del deterioro de } \\
\text { las condiciones climáticas. }\end{array}$ \\
\hline Objetivos & $\begin{array}{l}\text { Mejora de la } \\
\text { comercialización y de la } \\
\text { situación competitiva de } \\
\text { la instalación. } \\
\text { Clientes más } \\
\text { complacidos y fieles a la } \\
\text { instalación. } \\
\text { Disminución de costos de } \\
\text { no calidad debido a las } \\
\text { compensaciones. }\end{array}$ & $\begin{array}{l}\text { Indicadores de } \\
\text { competitividad. } \\
\text { Indicadores de calidad. } \\
\text { Cantidad de quejas de los } \\
\text { clientes. }\end{array}$ & $\begin{array}{l}\text { Libro de quejas de } \\
\text { clientes. } \\
\text { Encuestas a Clientes. }\end{array}$ & $\begin{array}{l}\text { Incremento del deterioro de } \\
\text { las condiciones de la } \\
\text { instalación. } \\
\text { Dificultades para la puesta } \\
\text { en práctica de estrategias } \\
\text { comerciales. }\end{array}$ \\
\hline Resultados & $\begin{array}{l}\text { Aumento de los niveles de } \\
\text { comercialización de la } \\
\text { instalación. } \\
\text { Mejora sustancial de la } \\
\text { competitividad comercial. }\end{array}$ & $\begin{array}{l}\text { Comportamiento de la } \\
\text { competencia. } \\
\text { Nivel de ocupación } \\
\text { mensual. } \\
\text { Nivel de la demanda actual } \\
\text { y futura. }\end{array}$ & $\begin{array}{l}\text { Balance Comercial. } \\
\text { Información de } \\
\text { comercialización por } \\
\text { ofertas. }\end{array}$ & $\begin{array}{l}\text { Aumento del deterioro de } \\
\text { las instalaciones, ofertas y } \\
\text { servicios de la entidad. } \\
\text { Aumento del deterioro de } \\
\text { las condiciones climáticas. }\end{array}$ \\
\hline Insumos & $\begin{array}{l}\text { Financiamiento para: } \\
\text { - Mejora de las } \\
\text { instalaciones del hotel. } \\
\text { - Promoción del } \\
\text { producto. } \\
\text { - Aplicación } \\
\text { estrategias } \\
\text { Marketing. }\end{array}$ & $\begin{array}{l}\text { Compras de suministros } \\
\text { para habitaciones y áreas } \\
\text { del hotel. } \\
\text { Campaña publicitaria y } \\
\text { promocional. }\end{array}$ & $\begin{array}{l}\text { Facturación de compras } \\
\text { y estado de los pagos } \\
\text { (balance económico). } \\
\text { Balance Comercial. }\end{array}$ & $\begin{array}{l}\text { Problema económico o } \\
\text { crisis financiera en el país, } \\
\text { turismo y la cadena hotelera. }\end{array}$ \\
\hline
\end{tabular}

Fuente: Elaboración propia. 


\section{Estructura organizativa del proyecto:}

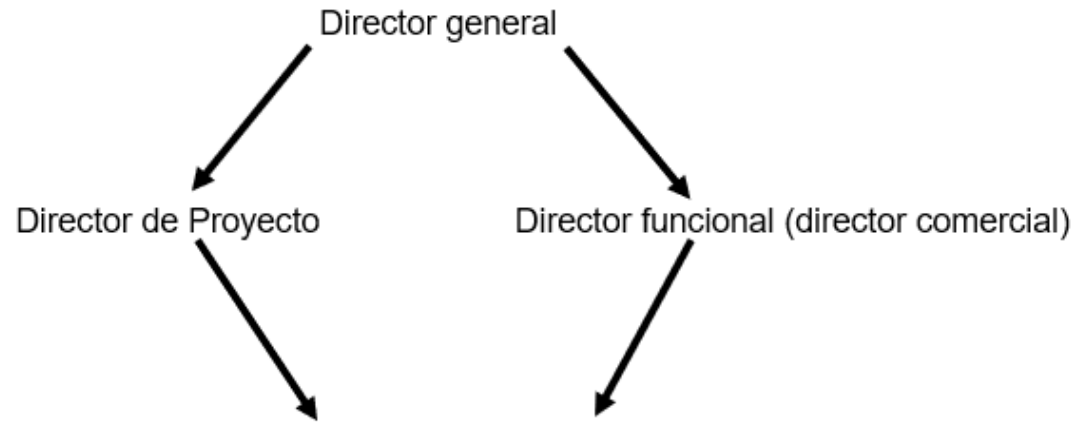

Equipo de proyecto

\section{Estructura fraccionada del trabajo}

\begin{tabular}{|c|c|c|c|c|c|c|}
\hline & ( & Nombre de tarea & Duración - & Comienzo & Fin & Predecesoras \\
\hline 1 & & $\begin{array}{l}\text { Diagnóstico de la } \\
\text { situación actual del } \\
\text { hotel. }\end{array}$ & 2 días & Iun $16 / 11 / 09$ & $\operatorname{mar} 17 / 11 / 09$ & \\
\hline 2 & & $\begin{array}{l}\text { Identificar los } \\
\text { principales problemas } \\
\text { del área comercial }\end{array}$ & 4 dias & mié 18/11/09 & lun $23 / 11 / 09$ & 1 \\
\hline 3 & & $\begin{array}{l}\text { Estudio de la situación } \\
\text { competitiva }\end{array}$ & 3 dias & mar 24/11/09 & jue $26 / 11 / 09$ & 2 \\
\hline 4 & & $\begin{array}{l}\text { Estudio de } \\
\text { prefactibilidad }\end{array}$ & 2 dias & vie $27 / 11 / 09$ & lun $30 / 11 / 09$ & $1 ; 2 ; 3$ \\
\hline 5 & & $\begin{array}{l}\text { Elaboración del } \\
\text { Proyecto }\end{array}$ & 10 dias & mar 01/12/09 & Iun $14 / 12 / 09$ & $1 ; 2 ; 3 ; 4$ \\
\hline 6 & & $\begin{array}{l}\text { Presentación del } \\
\text { proyecto }\end{array}$ & 1 día & $\operatorname{mar} 15 / 12 / 09$ & $\operatorname{mar} 15 / 12 / 09$ & 5 \\
\hline & & & & & & \\
\hline & & & & & & \\
\hline
\end{tabular}

\section{Diagrama de Gantt}

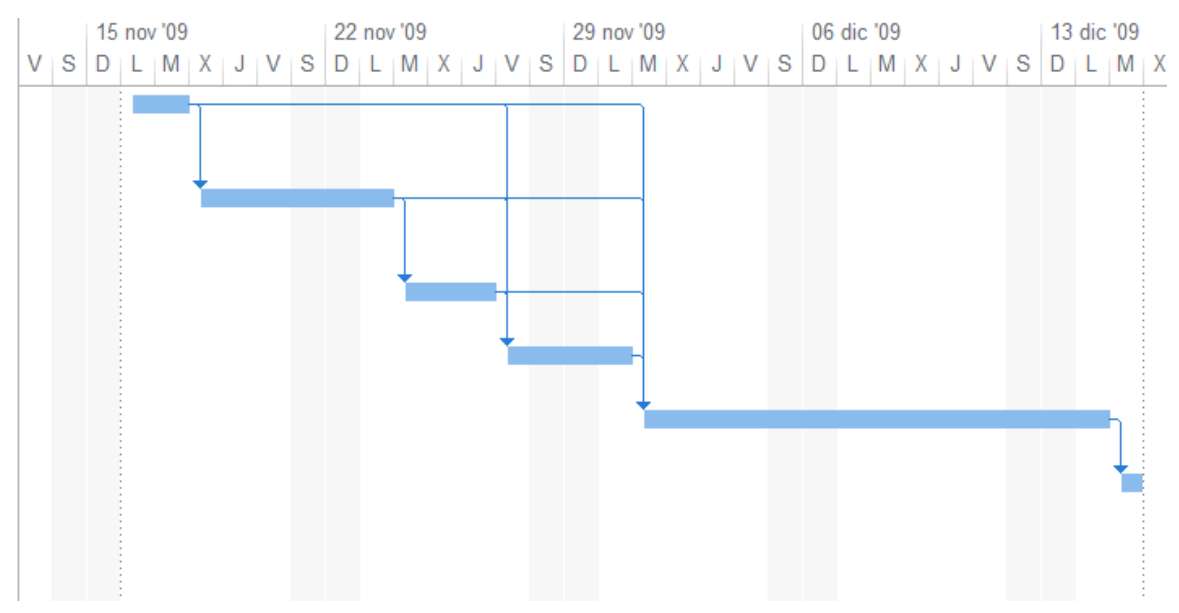




\section{Diagrama de Redes}

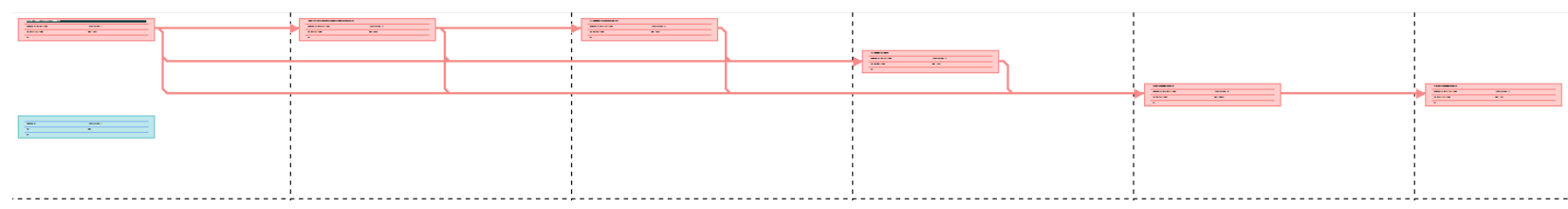

\section{Matriz funciones/Responsabilidades}

\begin{tabular}{|c|c|c|c|c|c|c|}
\hline $\begin{array}{c}\text { Función/ } \\
\text { Responsabilidad }\end{array}$ & $\begin{array}{l}\text { Equipo de } \\
\text { Proyecto }\end{array}$ & $\begin{array}{l}\text { Director de } \\
\text { Proyecto }\end{array}$ & $\begin{array}{l}\text { Turoperadores } \\
\text { y Agencias de } \\
\text { Viajes }\end{array}$ & MINTUR & $\begin{array}{l}\text { Gran } \\
\text { Caribe }\end{array}$ & Barceló \\
\hline $\begin{array}{c}\text { Diagnóstico de la } \\
\text { situación actual del } \\
\text { hotel. }\end{array}$ & & & + & + & + & + \\
\hline $\begin{array}{c}\text { Identificar los } \\
\text { principales problemas } \\
\text { del área comercial }\end{array}$ & & & + & & & \\
\hline $\begin{array}{l}\text { Estudio de la situación } \\
\text { competitiva }\end{array}$ & & & + & + & & \\
\hline $\begin{array}{c}\text { Estudio de } \\
\text { prefactibilidad }\end{array}$ & & & + & + & & \\
\hline $\begin{array}{l}\text { Elaboración del } \\
\text { Proyecto }\end{array}$ & & & + & + & & \\
\hline $\begin{array}{c}\text { Presentación del } \\
\text { Proyecto Final }\end{array}$ & & & & & & \\
\hline
\end{tabular}

Leyenda:

O: Elabora o ejecuta

$\mathrm{X}$ : Aprueba

$\triangle$ : Debe ser informado

+: Suministra la información

\section{Plan de mejoras:}

\section{Estrategias.}

Estrategias de producto/ servicio:

- Redefinición del portafolio de productos y servicios; coordinar con los Comités Organizadores, agencias receptivas y firmas extranjeras la inclusión del complejo como parte de los alojamientos de grupos de combinados y viajes de incentivos.

Estrategias de precio: 
- Establecer precios en contratos y ofertas de fácil reconocimiento por los clientes que los motive a consumir el producto.

Estrategias de distribución:

- Establecer contratos con los principales TT. OO de Canadá, Francia e Rusia.

Estrategias de comunicación:

- Impulsar la promoción en las redes sociales para mantener y mejorar la imagen favorable ante los grupos objetivos y captar la atención de los potenciales.

\section{Acciones a desarrollar}

- Realizar una correcta orientación al mercado que promueva la comprensión y gestión de información sobre los clientes de la empresa, competidores y fuerzas del entorno, con la finalidad de crear y mantener una oferta que genere mayor valor.

- Tener presente la revisión periódica y actualización del plan de marketing para asegurar la toma de decisiones comerciales con un enfoque sistemático y a la vez facilitar la ejecución de las acciones.

- Tener en cuenta estudiar el nicho de negocios y también la competencia antes de definir metas.

- Realizar un adecuado estudio de la competencia para poder establecer acciones que permitan diferenciar el servicio propio, del ofrecido por los competidores y de esta manera lograr ventajas competitivas sostenibles en el tiempo.

- Elaborar una estrategia de marketing en Internet teniendo como base la página web del hotel y las redes sociales, que juegan un papel importante en afianzar las relaciones directas con el cliente final.

- Introducir nuevas acciones de promoción y publicidad orientadas a captar aquellos mercados potenciales que más beneficios reporten al hotel. 


\section{Conclusiones.}

- La Dirección Integrada de proyectos permite desarrollar un estricto control de la puesta en práctica, progreso y adaptación del proyecto al contexto en el que este se lleva a cabo, lo cual favorece la disminución de los riegos y el alcance de los objetivos.

- El alcance de un eficiente nivel de competitividad comercial depende de un adecuado posicionamiento, correctas decisiones sobre producto/servicio, ventajosos precios, y apropiados canales de distribución y comunicación.

- La puesta en práctica de un proyecto vinculado a la competitividad comercial en el hotel Allegro Palma Real, contribuye a elevar el nivel competitivo de la instalación a través de herramientas más eficientes.

\section{Referencias bibliográficas.}

ABC, Definición. 2018. Definición de Competitividad empresarial. [En línea] 2018. $\mathrm{http}: / / \mathrm{ABC}$ definiciones/competitividad-comercial.com

Clelan, D., y Ring, W. Manual para la Administración de Proyectos. Mexico, 1993.

Domínguez Samá, O. 2018. Estrategias para elevar la competitividad comercial del hotel Allegro Palma Real. FORMATUR. Matanzas : s.n., 2018.

Heredia, Rafael. 1995. Dirección Integrada de Proyecto - Project Management - . 1995.

Hernández, Mauricio. 2016. La competitividad comercial. [En línea] 2016. https://prezi.com/mqufpobm_vyw/la-competitividad-comercial/ -->.

Méndez, Rafael. 2015. Formulación y evaluación de proyectos: enfoque para emprendedores. [En línea] 2017. https://Formulación y evaluación de proyectos_ enfoque para emprendedores - Google Libros_files/cb=gapi.loaded_0".

Polaino de los Santos, C. Introducción a la Gestión de Proyectos. s.l. : CETDIR-ISPJAE.

Rodríguez, Lourdes y Espinet, salvador. Dirección Integrada de Proyecto - Project Management. Folleto de apuntes - . 2002. 
S.A.2018. Competitividad Comercial. Qué necesita México para ser más competitivo. [En línea] Edacción/El empresario, 2018. http://elempresario.mx/competitividadcomercial -->.

Sapag, Nassir y Sapag, Reinaldo. 2010. Preparación y evaluación de proyectos. 2010.

Zamora Tabares, D, Alvarez García, P y Acosta Díaz, W. 2009. Gestión y Evaluación de proyectos Turísticos. 2009. 


\section{PARA CITAR EL ARTÍCULO INDEXADO.}

Manzano Coello, E. V., \& Padilla Martínez, M. P. (2020). La educación empresarial en gestión de operaciones integrada al desarrollo de emprendimientos innovadores. Visionario Digital, 4(4), 32-47. https://doi.org/10.33262/visionariodigital.v4i4.1417

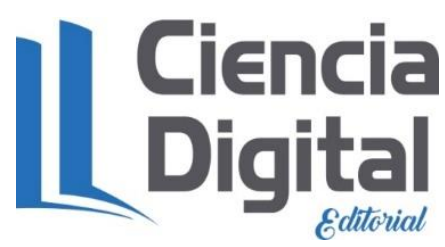

El artículo que se publica es de exclusiva responsabilidad de los autores y no necesariamente reflejan el pensamiento de la Revista Visionario Digital.

El artículo queda en propiedad de la revista y, por tanto, su publicación parcial y/o total en otro medio tiene que ser autorizado por el director de la Revista Visionario Digital.
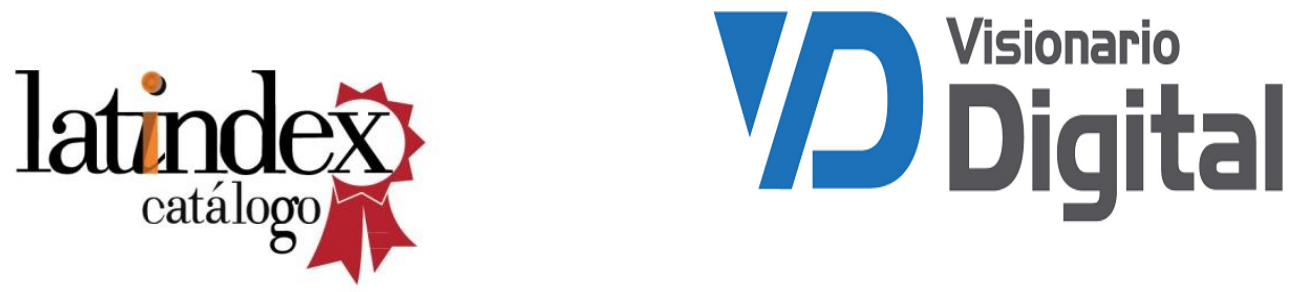